\title{
Numerical Investigation of Pulsations in a Plasma Arc Column
}

D. SCHAFFER ${ }^{1}$, R. TOUKHVATOULline, G. FELDMANN, Universidade Regional do Noroeste do Estado do Rio Grande do Sul - UNIJUí, Mestrado em Modelagem Matemática, Cx.P. 560, 98700-000 Ijuí, RS, Brasil.

\begin{abstract}
We investigate spatial fluctuations in a cylindrical plasma arc column in the interval of electric current $I=100-200 A$ and gas flow $G=4-8 \mathrm{~g} / \mathrm{s}$. The distribution functions of these oscillations were obtained. On the basis of experimental results we proposed a model for the description of the fluctuations. In this model the axis of the arc $\mathrm{O}^{\prime}$ participates at the same time on two movements: random oscillation and axial rotation. The lateral and radial intensities and radial temperatures were calculated for different rotation radii and standard deviations. For this study the spectral line of Ar I 415,8 $\mathrm{nm}$ was chosen. Experimental and theoretical results are in good agreement.
\end{abstract}

\section{Introduction}

As a rule, the radial temperature is determined by spectrometric methods such as the absolute line intensity and the relative line intensity [1]. With the absolute line intensity method, first the lateral intensity $I(x)$ is determined experimentally. After that the radial intensities and temperatures are determined solving the integral equation of Abel, knowing the relationship between the intensity and temperature. This procedure leads to good results when the plasma torch is stationary. But the experimental results show that the sources in plasma generators are not stationary. The electric current, tension, radiation intensity and temperature, as well as gas flow, velocity and the electric arc, oscillate [2-7]. In [4] studied the fluctuations of velocity, arc voltage and optical signal in a DC plasma torch. They found fluctuations of about $15 \%$ in these parameters. In Inductively Coupled Plasma (ICP) torch are hydrodynamic instabilities, due to the interaction of the plasma with cold atoms acting as heat sinks [5]. As these atoms present a near-turbulent movement, this interaction has a probability to occur in the whole plasma, rather than only in the injection region. The result of the study on pulsations in ICP plasma torch [6] show that it is almost free of spatial pulsations, but the pulsations in the radiation intensity due to the high frequency electric current exist. These pulsations are almost harmonic, superimposed by random oscillations. The amplitude of these pulsations reaches $10 \%$. In [7] it was studied the spatial and radiation intensity fluctuations of the vortex stabilized electric arc in the plasma generator. The study

\footnotetext{
${ }^{1}$ decio.schaffer@bol.com.br
} 
showed that for small gas flow rate these pulsations follow a Gaussian distribution, with relative standard deviation varying from 7 to $38 \%$.

These pulsations can affect the precision of experimental results. In [3], the influence of the frequency on the temperature of ICP torch was studied. It was shown that with an increase on frequency, the temperature in the axis decreases. In $[8,9]$ we studied the influence of the spatial and intensity pulsations on the radiation intensity and on the radial temperature profile on the supposition that they follow a Gaussian distribution. The results show that the pulsations of the plasma can significantly influence the experimental data and can explain the differences between the theoretical calculations and experimental measurements.

In the present work we studied ourselves more detailed the spatial oscillations on the stabilized electric arc aiming to find the arc axis distribution functions in the wide interval of gas flow and electric current. Based on the experimental results, a mathematical model of the cylindrical plasma is proposed, and the influence of pulsations on the radial temperature is calculated.

\section{Experimental investigation}

The experimental outline of the plasma generator is presented in Figure 1. The plasma generator consists of cathode (1), anode (2), coolant channels (3) and vortex chamber (4). The diameter of the arc channel is $1 \mathrm{~cm}$ and the distance between cathode and anode is $10 \mathrm{~cm}$. The working gas which produces vortex stabilization of the arc (6) enters tangentially at the anode through small orifices (5). The deviation of the arc at the $\mathrm{yOz}$ plan was registered on a film installed in a rotating chamber (7). The spatial pulsations of DC arc in the plasma generator for the range of current $I=100-200 \mathrm{~A}$ and gas flow $G=4-8 \mathrm{~g} / \mathrm{s}$ were studied. The results show that distribution functions were not Gaussian. A typical behavior of the electric arc oscillations for $z=8,5 \mathrm{~cm}, I=200 A$ and $G=6 \mathrm{~g} / \mathrm{s}$ is presented in Figure 1b. It is possible to observe quasi-harmonic oscillations superimposed by random oscillations.

The frequency of these pulsations varies between 9400 and $12400 \mathrm{~Hz}$ and depends weakly on the plasma gas flow rate and the current intensity. This quasiharmonic pulsations may be associated with the rotation of the arc around its center. The experimental results [2] are in favor of this conclusion, as it was shown that the electric arc in the gas flow has a spiral form. This form of the arc explains that the area of the minimum pressure is distributes along the spiral line.

The treatment of the experimental results of the electric arc oscillations have allowed us to build the arc axis distribution functions. In Figure 2 the distribution functions for the range of current $I=100-200 A$ and gas flow $G=4-8 \mathrm{~g} / \mathrm{s}$ are represented. It can be seen that as the current increases the area of oscillations also increases. This can be explained by the deviation of the electric arc under influence of the own magnetic field, which intensity increases with the increase in current intensity. Bases on these experimental results, a model of the nonstationary cylindrical plasma arc was proposed. Both types of pulsations occurring simultaneously in the plasma are considered in this model. The arc axis position 

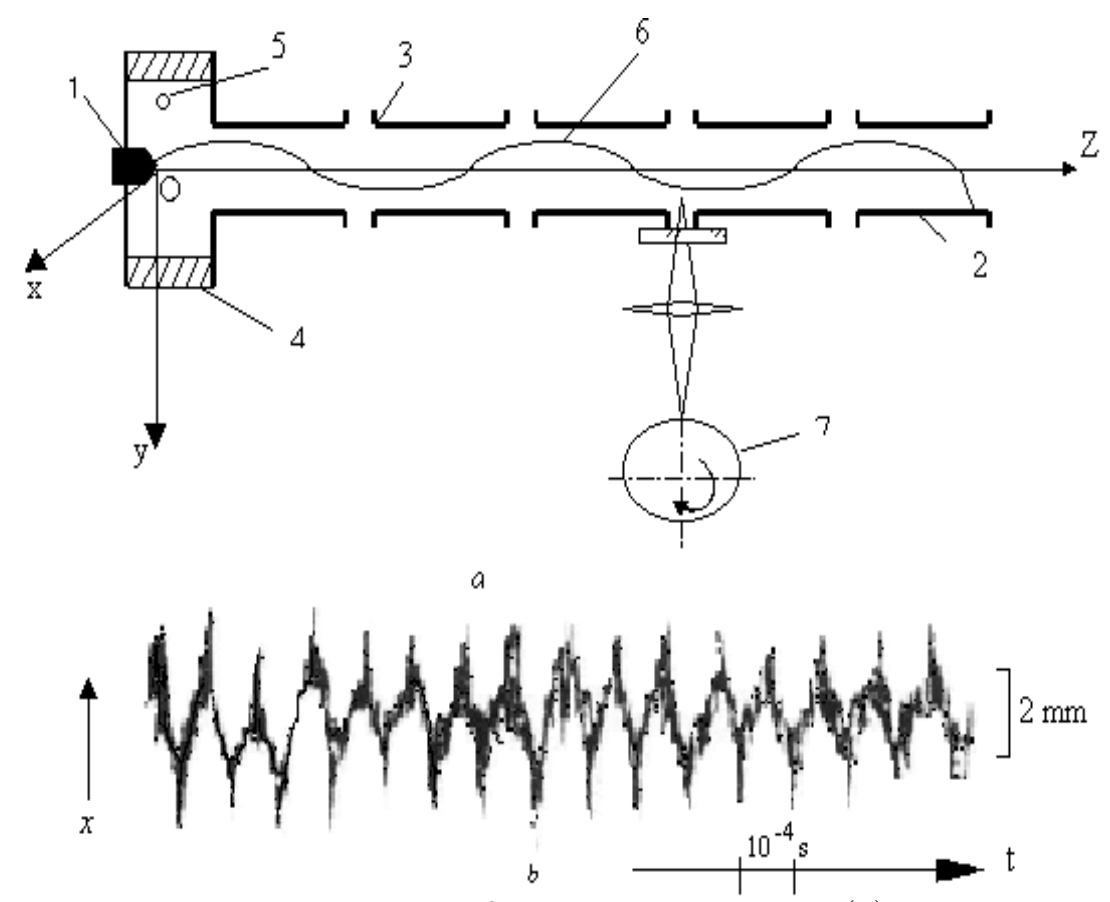

Figure 1: The experimental outline of the plasma generator $(a)$ and a typical behavior of the electric arc oscillations for $z=8.5 \mathrm{~cm}, I=200$ A and $G=6 \mathrm{~g} / \mathrm{s}$ (b).

describes the spatial pulsations around its center, which itself pulsates describing a circular motion.
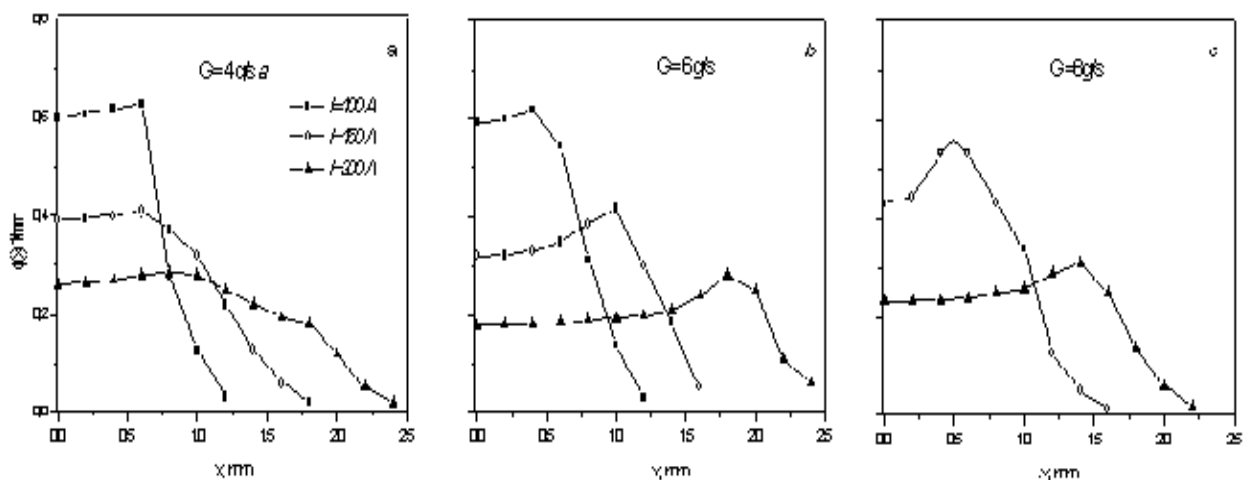

Figure 2: The experimental distribution functions $\varphi(x)$ for $\mathrm{G}=4 \mathrm{~g} / \mathrm{s}(a), 6 \mathrm{~g} / \mathrm{s}(\mathrm{b})$ and $8 \mathrm{~g} / \mathrm{s}(\mathrm{c}), I=100 A(\mathbf{\square}), \mathrm{I}=150 A(\mathrm{o})$ and $\mathrm{I}=200 A(\boldsymbol{\Delta})$. 


\section{Description of the model}

In present model we suppose the presence of two types of coupled pulsations, as shown in Figure 3. The axis $\mathrm{O}^{\prime}$ of the arc of radius $\mathrm{R}$ describes in the, $\mathrm{z}=$ constant plan, a circular motion around the axis $\mathrm{O}$ with radius $a$ in the laboratory coordinate system. The axis $\mathrm{O}^{\prime}$ also describes spatial pulsations following a Gaussian distribution

$$
\varphi_{1}(x)=\frac{1}{\sigma \sqrt{2 \pi}} \exp \left(-\frac{x^{2}}{2 \sigma^{2}}\right),
$$

where $\sigma$ is the standard-deviation. The projection of the circular motion of $\mathrm{O}^{\prime}$ around $\mathrm{O}$ on the $x O z$ plan oscillates according to

$$
x=a \sin \omega t,
$$

where $a$ is the rotation radius, $\omega$ is the angular frequency.

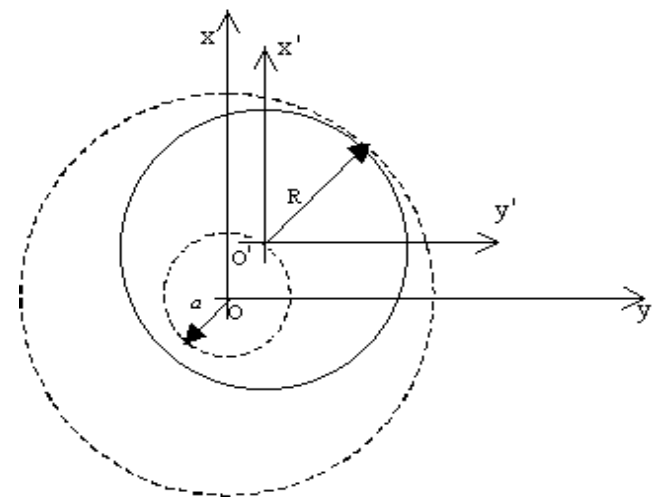

Figure 3: Scheme of the oscillations of the plasma arc; $x O y$ is the laboratory system and $x^{\prime} O^{\prime} y^{\prime}$ is the arc coordinate system.

The distribution function of these harmonic oscillations (3.2) is

$$
\varphi_{2}(x)=\frac{1}{\pi \sqrt{a^{2}-x^{2}}}, \quad-a \leq x \leq a .
$$

The total distribution function, which takes into account both types of pulsations can be expressed as:

$$
\varphi(x)=\int_{-a}^{+a} \varphi_{1}(x-\xi) \varphi_{2}(\xi) d \xi
$$

or, by substitution of equations (3.1) and (3.3),

$$
\varphi(x)=\int_{-a}^{+a} \frac{1}{\pi \sigma \sqrt{2 \pi\left(a^{2}-\xi^{2}\right)}} \exp \left[-\frac{(x-\xi)^{2}}{2 \sigma^{2}}\right] d \xi .
$$

Calculation of

$$
f=\int_{-\infty}^{+\infty} \varphi(x) d x
$$


changing the integration order, implies

$$
f=\int_{-a}^{+a} \frac{d \xi}{\pi \sqrt{a^{2}-\xi^{2}}} \int_{-\infty}^{+\infty} \frac{1}{\sigma \sqrt{2 \pi}} \exp \left[-\frac{(x-\xi)^{2}}{2 \sigma^{2}}\right] d x
$$

and, with consideration of the well-known integral

$$
\int_{-\infty}^{+\infty} \exp \left(-y^{2}\right) d y=\sqrt{\pi}
$$

leads to

$$
f=\int_{-a}^{+a} \frac{d \xi}{\pi \sqrt{a^{2}-\xi^{2}}}=1 .
$$

This means that for introduced function (3.5) the condition of the normalization is satisfied. In the limit case $\sigma \rightarrow 0$ we have

$$
\lim \frac{1}{\sigma \sqrt{2 \pi}} \exp \left[-\frac{(x-\xi)^{2}}{2 \sigma^{2}}\right]=\delta(x-\xi)
$$

and, respectively, $\varphi(x)=\varphi_{2}(x)$. In the other limit case when $a \rightarrow 0$, as it can be seen from Eq. (3.5), $\varphi(x)=\varphi_{1}(x)$. Then, the equation (3.5) generalizes the functions (3.1) e (3.3).

With introduction of the dimensionless quantities $\alpha=x / a ; \beta=\xi / a ; \zeta=\frac{\sigma}{a}$; $\psi(a)=\pi a \varphi(x)$ the Eq. (3.5) can be rewritten as follows:

$$
\psi(\alpha)=\frac{1}{\zeta \sqrt{2 \pi}} \int_{-1}^{1} \frac{1}{\sqrt{\left(1-\beta^{2}\right)}} \exp \left[-\frac{(\alpha-\beta)^{2}}{2 \zeta^{2}}\right] d \beta .
$$

It can be seen on this relationship that $\psi(\alpha)$ depends only of $\zeta$. This relation $\psi(\alpha)$ is presented in Figure 4.

The curve 4 corresponds the case $\zeta=0$ (random oscillations absent) and it is determined by the relationship

$$
\psi(\alpha)=\frac{1}{\sqrt{1-\alpha^{2}}} .
$$

Curves 1 and 2 refer to $\zeta=0.2$ e 0.4 respectively. The dependence $\psi(0)$ on $\zeta$ is shown by curve 3 . It can be observed that on the axis the function $\psi(0)$ increases with $\zeta$, it reaching a maximum and later decreasing again.

Considering the relationships

$$
\bar{\varphi}=\frac{\varphi_{\max }}{\varphi(0)}, \quad \bar{\psi}=\frac{\psi_{\max }}{\psi(0)} \quad \text { and } \quad \bar{\varphi}=\bar{\psi}
$$

we can find $\zeta$ from the illustrations 2 and 4 (curve 5). The theoretical dependence of $\bar{\psi}(\zeta)$ in Figure 4 is presented by the curve 5 . The values $a$ and $\sigma$ are found from the relationships $a=\frac{\psi(0)}{\pi \varphi(0)}$ and $\sigma=\zeta a$. 


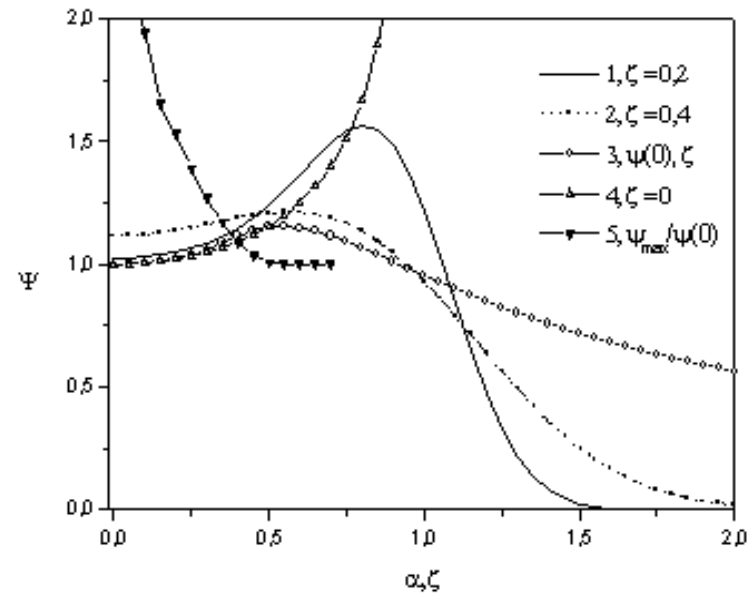

Figure 4: The theoretical dependence $\psi(\alpha) .1-\zeta=0.2,2-\zeta=0.4,3-\psi(0)$ of $\zeta, 4-\zeta=0,5-\bar{\psi}(\zeta)$.

Table 1: Data for $\zeta, a$ and $\sigma$ as function of $G$ and $I$.

\begin{tabular}{|c|c|c|c|c|c|c|c|c|c|}
\hline & $\begin{array}{c}G, \\
g / s\end{array}$ & $I, A$ & $\begin{array}{c}\varphi_{\max }, \\
1 / \mathrm{mm}\end{array}$ & $\begin{array}{c}\varphi(0), \\
1 / m m\end{array}$ & $\bar{\varphi}_{\max }$ & $\zeta$ & $\psi(0)$ & $\begin{array}{c}\mathrm{a}, \\
\mathrm{mm}\end{array}$ & $\begin{array}{c}\sigma, \\
\mathrm{mm}\end{array}$ \\
\hline 1 & 4 & 100 & 0,63 & 0,6 & 1,05 & 0,47 & 1,144 & 0,607 & 0,285 \\
\hline 2 & 4 & 150 & 0,41 & 0,39 & 1,03 & 0,46 & 1,14 & 0,931 & 0,428 \\
\hline 3 & 4 & 200 & 0,285 & 0,26 & 1,115 & 0,38 & 1,105 & 1,354 & 0,515 \\
\hline 4 & 6 & 100 & 0,62 & 0,59 & 1,034 & 0,47 & 1,14 & 0,615 & 0,289 \\
\hline 5 & 6 & 150 & 0,415 & 0,32 & 1,297 & 0,285 & 1,055 & 1,05 & 0,299 \\
\hline 6 & 6 & 200 & 0,28 & 0,18 & 1,56 & 0,19 & 1,02 & 1,805 & 0,343 \\
\hline 7 & 8 & 150 & 0,535 & 0,43 & 1,244 & 0,31 & 1,07 & 0,794 & 0,246 \\
\hline 8 & 8 & 200 & 0,31 & 0,235 & 1,292 & 0,29 & 1,055 & 1,43 & 0,425 \\
\hline
\end{tabular}

Data for of $\zeta, a$ and $\sigma$ are presented in Table 1 . The table shows that, with increase of current and decrease of gas flow, the rotation radius of the electric arc and the standard-deviation $\sigma$ increase.

The comparison of experimental and theoretical results are presented in Figure 5. In this illustration the continuous curves are theoretical (build up by 3.6). Isolated points represent experimental data. The numbers associated to the symbols correspond to the numbered lines on Table 1. It can be seen from this figure, that the distribution function $\psi(\alpha)$ depends only on $\zeta$ and that equation (3.6) correctly describes the pulsations in the plasma generator. This corroborates the proposed model. 


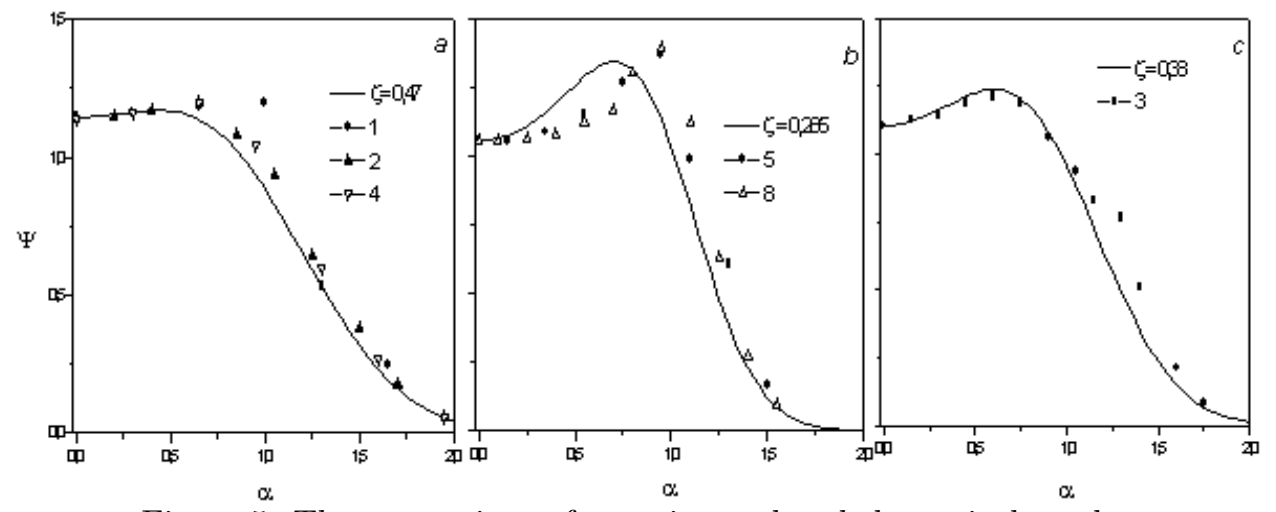

Figure 5: The comparison of experimental and theoretical results.

\section{Intensity and Temperature of the Oscillating Plasma}

The lateral intensity $I(x)$ of observed radiation by the spectrometer can be related to the intensity in the plasma system $q(x)$ by the Fredholm integral equation of the first kind

$$
I(x)=\int_{-R}^{R} q\left(x^{\prime}\right) \varphi\left(x-x^{\prime}\right) d x^{\prime} .
$$

Here $\varphi\left(x-x^{\prime}\right)$ is the distribution function associated with the oscillations of the torch axis described by (3.5). $\mathrm{R}$ is the radius of the plasma torch. In accordance to [8], we assume that the actual lateral intensity $I(x)$ can be described by:

$$
I(x)=I(0) \exp \left(-k x^{2}\right) .
$$

We solved the Fredholm's equation (4.1) for the Phillips - Twomey regularization method [10] using a program in language $C$. This equation was solved numerically for $\mathrm{k}=10, \mathrm{R}=0.5 \mathrm{~cm}, \sigma$ and $a=0.05,0.075,0.10 \mathrm{~cm}$. The results of the calculations of $q(x)$ for $k=10, \mathrm{R}=0.5 \mathrm{~cm}, \sigma$ and $a=0.05 \mathrm{~cm}$ are presented in Fig. (6).

It can be seen from this graph that the presence of oscillations reduces the intensity in the axis and it increases it in the periphery of the torch.

To find the radial temperatures, the radial intensities were calculated, solving Abel's integral equation:

$$
I(x)=2 \int_{x}^{R} \frac{\varepsilon(r) r d r}{\sqrt{r^{2}-x^{2}}}, 0 \leq r \leq R,
$$

where $\varepsilon(r)$ is the radial intensity. For the calculations of $\varepsilon(r)$ equation (4.3) was solved numerically [1]. The calculations of $\varepsilon(r)$ were performed for $\sigma$ and $a=0$, $0.05,0.1,0.015 \mathrm{~cm}$. The particular results of $\varepsilon(r)$ for $\sigma=0.05 \mathrm{~cm}, a=0.05 \mathrm{~cm}$ are presented in Figure 7. 


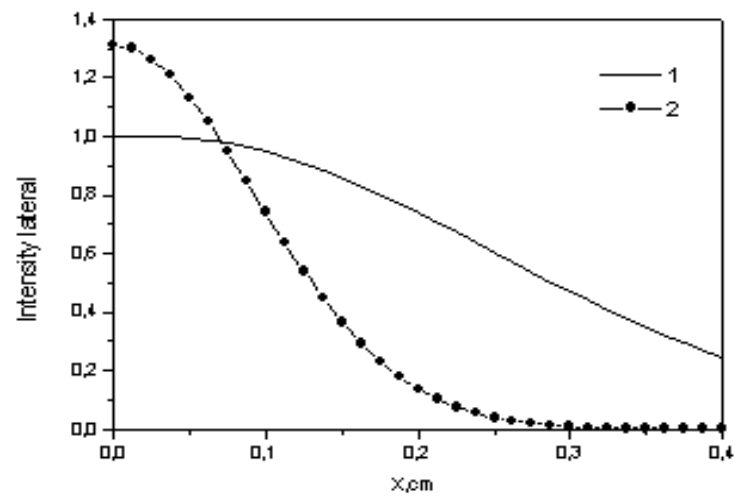

Figure 6: Relative lateral intensities in laboratory system (1) and in plasma system for $\sigma=0.05 \mathrm{~cm}$ and $a=0.05 \mathrm{~cm}(2)$.

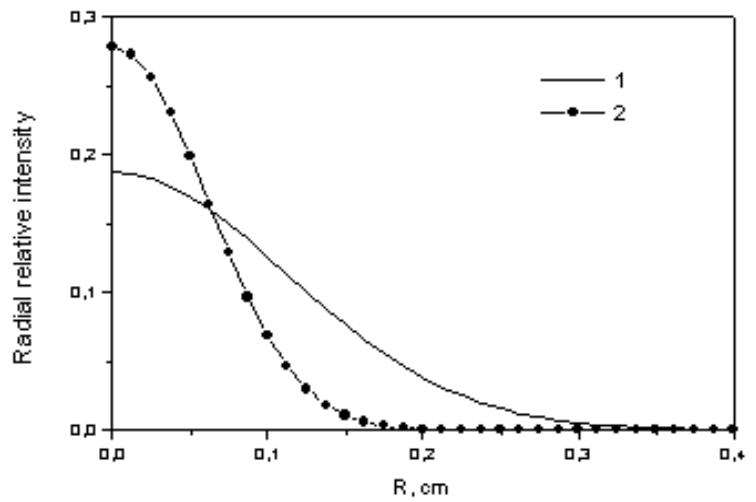

Figure 7: Relative radial intensities in laboratory system (1) and in plasma system for $\sigma=0.05 \mathrm{~cm}$ and $\mathrm{cm}$ and $a=0.05 \mathrm{~cm} \mathrm{(2).}$

To find the radial temperature of the plasma the following relationship is used which related the local intensity of a spectral line to the temperature [1]:

$$
\varepsilon_{\nu}=A_{n}^{m} \frac{g_{m}}{Z} n_{a} \exp \left(-\frac{E m}{k T}\right) h \nu,
$$

where $A_{n}^{m}$ is the radioactive decay probability from $m$ to $n$ energy levels, $E_{m}$ and $g_{m}$ are respectively the energy and statistical weight of level $m, Z$ is the partition function, $n_{a}$ is the concentration of the atoms, $k$ is Boltzmann's constant, $h$ is the Planck's constant and $\nu$ is the frequency.

We calculated the dependence (4.4) for the spectral line $A r I 415.8 \mathrm{~nm}$ in the interval of temperatures $3000-26000 \mathrm{~K}$ [8]. The graph of the relationship (4.4) in the interval of temperatures 8000 - $12000 \mathrm{~K}$ is presented in Figure 8.

The calculations of radial temperatures starting from radial intensities (Figure 7) and the dependence between the intensity and the temperature (Figure 8) were accomplished for two temperatures in the axis of the arc $T=10000 K$ and 12000 $K$. The calculations were made for the stationary plasma $(\sigma=0, a=0)$ and 


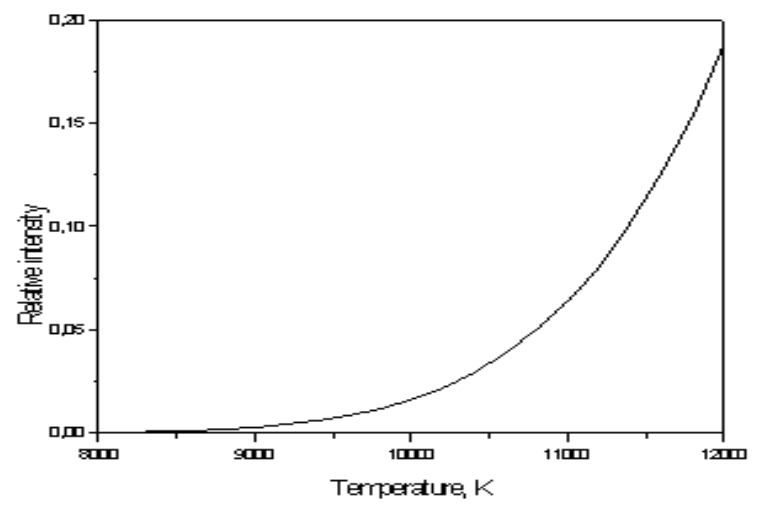

Figure 8: Relative intensity of the line $A r$ I $415.8 \mathrm{~nm}$ in function of the temperature.

not stationary plasma ( $\sigma$ and $a=0.05,0.1,0.15 \mathrm{~cm}$ ). Figure 9 presents radial temperatures for $\mathrm{T}=12000 \mathrm{~K}$ on the axis, $\sigma=0.05 \mathrm{~cm}, a=0.05$. The curve 1 in this illustration corresponds the radial temperature in plasma system. It should be noted from this figure that the pulsations exert considerable influence on the radial temperature. This influence depends on the radius of rotation $a$ and on the standard deviation $\sigma$.

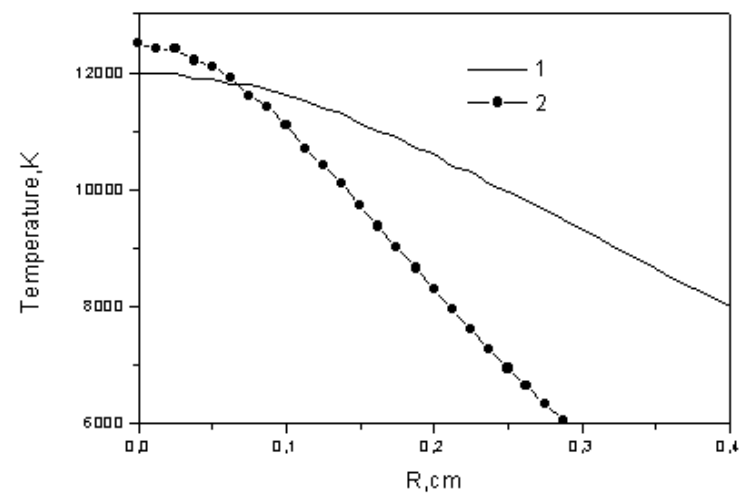

Figure 9: Radial distribution temperature in laboratory system (1) and in plasma system for $\sigma=0.05 \mathrm{~cm}$ and $a=0.05 \mathrm{~cm} \mathrm{(2).}$

\section{Analysis of Results and Conclusion}

The comparison of experimental and theoretical results presented in Figure 5 show that the theoretical distribution function equation (3.6) correctly describes the space fluctuations of the arc in the plasma generator. This means that the proposed model is satisfactory. It can be seen from Figures 6 and 9, that space pulsations of the electric arc provoke a decreasing of the line intensity and of the temperature at the axis of the arc as well as an enlargement of the profile and an increasing of the lateral distribution and temperature in the periphery. The shape of the curve 
is dependent of the combination of the parameters $\sigma$ and $a$. From results of this work we conclude that the plasma space pulsations can influence considerably in the precision of the determination of the radial temperature.

Resumo: Neste trabalho, foram investigadas flutuações em plasma de arco elétrico estabilizado com simetria cilíndrica, em intervalos de corrente elétrica $I=100-$ $200 A$ e de injeção de gás $G=6-8 \mathrm{~g} / \mathrm{s}$. A função de distribuição destas oscilações foi obtida a partir de dados experimentais. Foi proposto um modelo físico-matemático que descreve estas flutuações. Neste modelo o eixo do arco $O^{\prime}$ participa ao mesmo tempo de dois tipos de movimentos: oscilações randômicas e rotação axial. A partir deste modelo, a intensidade radial, lateral e a temperatura radial foram calculadas para diferentes raios de rotação e desvios padrões. Para este estudo foram utilizadas as linhas espectrais do Ar I 415,8 $\mathrm{nm}$. Os resultados experimentais e teóricos mostraram-se em boa concordância.

\section{References}

[1] S.V. Dresvin, "Physics and Technology of Low-Temperature Plasmas" (translation from Russian), Iowa State University Press, 1977.

[2] M. Joukov, V. Smoliakov and B. Uriukov, "Plazmotrons", Nauka, 1983.

[3] J. Mostaghimi and M.I. Boulos, J. Appl. Phys., 68 (1990), 2643-2648.

[4] M.P. Planche, J.F. Coudert and P. Fauchais, Plasma Chem. Plasma Process, 18 (1998), 263-283.

[5] P. Proulx, J. Mostaghimi and M.I. Boulos, Int. J. Heat Mass Transfer, 34 (1991), 2571-2579.

[6] R. Toukhvatoulline, H. Bonadiman and M. Pieniz, em "Seleta do XXII CNMAC", TEMA - Tendências em Matemática Aplicada e Computacional, Vol 1., pp. 223-232, SBMAC, 2000.

[7] R. Toukhvatoulline, "Investigation of radial distribution of the temperature in DC plasma torch", PhD Thesis Kazan Technical University", Kazan (in Russian), 1975.

[8] R. Toukhvatoulline and G. Feldmann, Fresenius J. Anal. Chem., 364 (1999), 517-520.

[9] R. Toukhvatoulline and G. Feldmann, J. Phys. D: Appl. Phys., 33 (2000), 2420-2424.

[10] W.H. Press et al., "Numerical Recipes in C. The art of scientific computing", Second edition, 1992. 